\title{
Equations of anomalous diffusion of cosmic ray
}

\section{Ilolov Mamadsho*, ${ }^{\dagger}$ Kuchakshoev Kholiknazar „‡ Puchkov Vitaly *, Yormamadov Shakarmamad $\star$}

$\dagger$ Center Of Innovative Development of Science and New Technologies, Academy of Sciences of the Republic of Tajikistan

33 Rudaki ave. Dushanbe 734025 Tajikistan

${ }^{\ddagger}$ Russian -Tajik(slavonic) University

30 Tursunzoda str. Dushnabe, 734025 Tajikistan

*Lebedev Physical Institute, Russian Academy of Sciences

53 Leninsky prospect Moscow 119991 Russia

"International Scientific Research Center "Pamir - Chakaltay"

209 Ainy str. Dushanbe 734018 Tajikistan

E-mail: ilolov.mamadsho@gmail.com, kholiknazar76@gmail.com,

puchkovesci.lebedev.ru,yormamadov@mail.ru

The problem of origin of ultra high energy cosmic rays (UHECR) was the subject of intensive investigations of last decades. Primarily, experimental and theoretical works touch upon the issues of Greisen - Zacepin - Kuzmin(GZK) effect. This paper is dedicated to mathematical modeling of propagation process of UHECR. It is prescribed that anomalous diffusion of particles in quasihomogenous medium we can describe with fractional partial differential equations. Central idea is derivation of fractional analogue of Fick's law.

The 34th International Cosmic Ray Conference

30 July- 6 August, 2015

The Hague, The Netherlands

\footnotetext{
* Speaker.
} 


\section{A. Introduction}

The problem of origin of ultra high energy cosmic rays (UHECR) was the subject of intensive investigations of last decades. Primarily, experimental and theoretical works touch upon the issues of Greisen - Zacepin - Kuzmin(GZK) effect. This paper is dedicated to mathematical modeling of propagation process of UHECR. It is prescribed that anomalous diffusion of particles in quasihomogenous medium we can describe with fractional partial differential equations. Central idea is derivation of fractional analogue of Fick's law.

\section{B. GZK problem (early and new results)}

\section{B.1}

In 1966 was offered the following hypothesis in papers K.Greisen [1] and G.T. Zacepin, V.A. Kuzmin [2]:

"Due to interactions with the relic microwave photons, protons (or nuclear) of cosmic rays have great energy losses, which should carry out a "cut - off " of the energy spectrum at energies (3 -6) $10^{19} \mathrm{eV}$, if we assume a uniform distribution of particles with such sources of energy in the Universe."

The process of photoproduction of pions in interactions with relic radiation significantly limits the possible distance to the sources for the Ultra high energy cosmic rays (UHECR), which is based on the GZK - effect cannot exceed 100 Mps. At this distances must occurs suppression of high-energy part of the spectrum.

It should be noted that the established in 70's under the leadership of G.T. Zatsepin installation titled "Experiment Pamir" (Eastern Pamirs, Tajikistan), opened the way to ultra-high energies. G.T. Zatsepin also developed a method of $\mathrm{X}$ - ray emulsion chambers for the detection of particles with energies $E \gtrsim 10^{12} \mathrm{eV}$ with a spatial resolution of about 300 microns. In those years, the maximum power that can be achieved using the accelerator, do not exceed $100 \mathrm{GeV}$. At the present time, despite the rapid development of collider technology (LHC), used the counter-rays colliding protons, the maximum energy is $1,6 \times 10^{15} \mathrm{eV}$, and in the Pamir experiment account the interaction with the energy is more than $10^{17} \mathrm{eV}[3,4]$.

High altitude experiments like "Pamir" are now becoming an important linkage between direct and indirect managements of UHECRs providing CR physicists with comprehensive information relevant to intermediate energy range $\left(10^{15}-10^{19} \mathrm{eV}\right)$ which contains both the "knee" and the "ankle" and where a connection the Galactic and extragalactic CR takes place.

Registered events in the last decades with energies exceeding the GZK - limit with energies on experiments SUGAR [5], Haverah Park [6], Yakutsk [7] and AGASA [8] and telescopes Fly's Eye [9] and HiRES [10] led to new unresolved problems that are formulated in [10] as follows:

1. In the spectrum UHECR is not GZK - suppression .

2. The direction of arrival of particles with energies $E>10^{20} \mathrm{eV}$ will not lead to the identification of astrophysical sources inside the GZK - sphere.

As a possible solution to the second part of the problem has been hypothesized that the sources are not UHECR unique astrophysical objects, but something that was (or is ) within the GZK - 
sphere, but today it is not visible and does not manifest itself as the source, except for the presence of luminosity in UHECR (" dead quasar " [11] ).

From March 2008 started working detectors of Telescope Array(TA) (Utah,USA) - the largest in the Northern Hemisphere installation studying UHECR. Found in addition to the " knee " $(\log (E[e V])=15)$, two more break points for values of $\log (E[e V])$ equal to 18.71 and 19.75 , which corresponds to " ankle " and the beginning of GZK - suppression. When energy exceeds the break point $E=10^{19,75} \mathrm{eV}$ registered five events, whereas a power extension of the continuous spectrum would be expected 18.4 events [12].

\section{B.2}

Anisotropy of UHECR flow. Investigation of UHECR will open a window into a highenergetic Universe. Measurements of anisotropy phenomena are one of the most important unsolved problems in physics of UHECR. The discovery of the anisotropy will shed light on the identification of UHECR. From the results of correlation analysis of the transition areas of the primary particles with the large-scale structure of the Universe implies that TA events occur in the sources that follow the distribution of matter in the Universe.

\section{Anomalous diffusion model}

\section{C.1}

Diffusion transfer theory of galactic cosmic rays is mainly developing in two directions - model of isotropic diffusion and model of anisotropic diffusion [13] . In paper [14] the process of movement of charged particles in interstellar magnetic fields decomposes into longitudinal and cross components in the form of compound diffusion. In paper [15] sub diffusion and super diffusion regimes are unified with equation

$$
\left[D_{t}^{\beta}+K(E)(-\Delta)^{\alpha / 2}\right] u(\vec{x}, E, t)=\delta(\vec{x}) \delta(E) \delta_{\beta}(t),
$$

where $0<\alpha \leq 2,0<\beta \leq 1, K$ - coefficient of diffusion depending on energy $E, \delta_{\beta}(t)$ - fractional generalization of delta function $\delta(t)$. Solution of equation (1) - fractional stable distribution with "heavy power tail" type of singularity in case $\alpha<2$, demonstrates special behavior in origin and corresponds to diffusion packet expansion with time $t^{\beta / \alpha}$.

Model developed on the basis of equation (1) is called model of anomalous diffusion of spreading galactic cosmic rays.

This model was used for explanation of fracture of energy spectrum of "knee" type i.e increasing of index $\eta$ in exponential representation $E^{-\eta}$ in case crossing from domain $\sim 10^{2} \mathrm{GeV} /$ nucleon to domain $10^{5} \mathrm{GeV} /$ nucleon.

\section{C.2}

In this paper possibility of galactic and intergalactic scenarios of UHECR derivation is accepted. Taking into account recent results received in different stations of cosmic rays on testing reliability of GZK hypotheses and with the use of fraction analogue of Fick's law it is offered new variant of UHECR spreading in inhomogeneous interstellar medium. 
Such model is based on separation to wide extent between small-scale mechanism of transportation of charged particles and large-scale process of variation of regular gradient of transferable substance. Mathematical description of such approach is connected with involvement of differential equations with fractional derivatives both in time and space.

For simplicity we restrict our consideration with one-dimensional diffusion process describable by probability density function $u(x, E, t)$. Then standard diffusion equation for $u(x, E, t)$ is

$$
\frac{\partial u(x, E, t)}{\partial t}=K(E) \frac{\partial^{2} u(x, E, t)}{\partial x^{2}}+S(x, E, t)
$$

where $K(E)>0$ - diffusion coefficient depending on energy $E$.

Solution $u(x, E, t)$ is scalar flux of particles of cosmic radiation with energy $E$ of sources distribution with density $S(x, E, t)$.

Appropriate homogenous equation $(S(x, E, t)=0)$ follows from continuity equation (conservation law)

$$
\frac{\partial u(x, E, t)}{\partial t}+\frac{\partial f(x, E, t)}{\partial x}=0,
$$

where $f(x, E, t)$ is flux defined by Fick's law

$$
f(x, E, t)=-K(E) \frac{\partial u(x, E, t)}{\partial x} .
$$

Relation (C.4) is the simplest phenomenological equation connecting particles flux of cosmic radiation and gradient of density.

It can be replaced by more common law, which allows adequate description of nonlinear process of transference. Generalized diffusion equation, where derivative with respect to time variable replaced by fractional derivative and second order derivative with respect to space variable replaced by pseudodifferential operator, can be adapted for modeling nonlocal effects of spread of cosmic rays. For the first time such type of equation was considered in paper [13]. Detailed analysis of such first order in time equations we can find in papers [15, 16].

We write appropriate equation for fractional diffusion in form

$$
\partial_{t}^{\beta} u(x, E, t)=K(E) D_{\theta}^{\alpha} u(x, E, t), K(E)>0,1<\alpha \leq 2,0<\beta \leq 1,|\theta| \leq 2-\alpha,
$$

where $\partial_{t}^{\beta}$ - Caputo fractional derivative of order $\beta$ given by formula

$$
\left(\partial_{t}^{\beta} u\right)(t)=\left(D_{0+}^{\beta} u\right)(t)-\frac{1}{\Gamma(1-\alpha)} \frac{u(x, E, 0)}{t^{\alpha}},
$$

and

$$
\left(D_{0+}^{\beta} u\right)(t)=\frac{1}{\Gamma(1-\alpha)} \frac{d}{d t} \int_{0}^{t} \frac{u(x, E, \tau)}{(t-\tau)^{\alpha}} d \tau
$$

is Rieman-Liouville fractional derivative [20]; pseudodifferential operator $D_{\theta}^{\alpha}$ is defined by formula

$$
\left(D_{\theta}^{\alpha} u_{0}\right)(x)=-\left[C_{+}(\alpha, \theta) D^{2} I_{+}^{2-\alpha}+C_{-}(\alpha, \theta) D^{2} I_{-}^{2-\alpha}\right] u_{0}(x) .
$$

Here $D^{2}=\frac{d^{2}}{d x^{2}}$ and $I_{ \pm}^{\gamma}(\gamma=2-\alpha)$ are Weyl integrals of order $\gamma$

$$
I_{+}^{\gamma} u_{0}(x)=\frac{1}{\Gamma(\gamma)} \int_{-\infty}^{x}(x-\xi)^{\gamma-1} u_{0}(\xi) d \xi, \quad I_{-}^{\gamma} u_{0}(x)=\frac{1}{\Gamma(\gamma)} \int_{x}^{\infty}(\xi-x)^{\gamma-1} u_{0}(\xi) d \xi
$$


Coefficients $C_{ \pm}$- appropriate weighting functions reducing to asymmetrical operator in case $\theta \neq 0$ :

$$
C_{ \pm}(\alpha, \theta)=\frac{\sin [(\alpha \pm \theta) \pi / 2]}{\sin (\alpha \pi)}
$$

It is obvious that $\alpha=2$ we can obtain when $\theta$ tends to zero and

$$
C_{+}(2,0)=C_{-}(2,0)=-1 / 2, D_{0}^{2}=D_{1}^{2} .
$$

Representation of stable probability density functions are given in papers [17, 18, 19] and has the form of convergent series for positive power of $\zeta>0$

$$
P_{\alpha}(\zeta, \theta) \sim \frac{1}{\pi \zeta} \sum_{n=1}^{\infty}(-\zeta)^{n} \frac{\Gamma(n / \alpha+1)}{n !} \sin \left[\frac{n \pi}{2 \alpha}(\theta-\alpha)\right], 1<\alpha \leq 2
$$

and divergent series for negative power of $\zeta>0$

$$
P_{\alpha}(\zeta, \theta) \sim \frac{1}{\pi \zeta} \sum_{n=1}^{\infty}\left(-\zeta^{-\alpha}\right)^{-n} \frac{\Gamma(n \alpha+1)}{n !} \sin \left[\frac{n \pi}{2}(\theta-\alpha)\right], 1<\alpha<2,
$$

where (C.11) exists for any $\theta$ satisfying condition $|\theta| \leq 2-\alpha$, and (C.9) does not exist for extreme negative value $\theta=\alpha-2$ for which appropriate density function demonstrates exponential destruction. For $\zeta<0$ there exists identity

$$
P_{\alpha}(-\zeta, \theta)=P_{\alpha}(\zeta,-\theta)
$$

It follows from (C.12), that for $\alpha \neq 2$ stable probability density function represent heavy tails failing as $|\zeta|^{-\alpha+1}$ at $|\zeta \rightarrow \infty|$ with the exception of two extremal cases $\theta=\mp(2-\alpha)$ for which the tail at $\zeta \rightarrow \pm \infty$ vanishes exponentially. In case $\alpha=2(\theta=0)$ from (C.11) we obtain Gaussian function

$$
P_{2}(\zeta, 0)=\frac{1}{2 \sqrt{\pi}} \exp \left(-\zeta^{2} / 4\right)
$$

Pseudodifferential operator $D_{\theta}^{\alpha}$ defined in (C.5) is a Feller fractional derivative of order $\alpha$ and asymmetry of $\theta$.

It is well known [19] that generating pseudodifferential operator $A$ acting on variable $x \in R$ is determined through its Fourier transform, exactly

$$
\hat{A} \varphi(k, t)=\hat{A}(k) \hat{\varphi}(k, t),
$$

where $\hat{A}(k)$ determines symbol of operator $A$.

Operator of $n$ times differentiation $D^{n}=\frac{\partial^{n}}{\partial x^{n}}$ is a special case with symbol $\hat{D}^{n}=(-i k)^{n}$.

Generally pseudodifferential operator $A$ defines with the aid of kernel of spacial integral of convolution. This kernel is absolutely integrable function on $R$, which degenerates into distribution of delta function type in case $A=D^{n}$. Symbol of operator $A$ is given through following rule

$$
\hat{A}(k)=\left(A e^{-i k x}\right) e^{-i k x} \text {. }
$$

Now we can write new relation between flux and gradient of density function. In other words we can write relation which generalizes Fick's law (C.3) on pseudodifferential operator depending on $\alpha$ and $\theta$. 
After series of simplifications we obtain the equation

$$
f(x, E, t)=P_{\theta}^{\alpha}\left[-\frac{\partial u(x, E, t)}{\partial x}\right]
$$

where $P_{\theta}^{\alpha}$ is a pseudodifferential operator with symbol

$$
\hat{P}_{\theta}^{\alpha}(k)=\frac{\hat{D}_{\theta}^{\alpha}(k)}{\hat{D}^{2}(k)}=|k|^{\alpha-2} e^{i(s i g n k) \theta \pi / 2},
$$

For $\alpha=2$ we obtain standard Fick's law since in this case $P_{0}^{2}=I$ ( $I$ - is unit operator).

For $1<\alpha<2$ equation (C.13) establish space nonlocal connection between flux and gradient of density function. It should be noticed that for $0<\alpha \leq 1$ equation (C.13) is meaningless, since in (C.14) symbol of pseudodifferential operator $P_{\theta}^{\alpha}$ has singularity at $k=0$. It means that appropriate kernel is not integrable in $R$.

Above mentioned difficulty we can overcome using another relation between flux and density function of type

$$
f(x, E, t)=Q_{\alpha}^{\theta} u(x, E, t),
$$

where $Q_{\alpha}^{\theta}$ is another operator with symbol

$$
\hat{Q}_{\theta}^{\alpha}(k)=-\hat{P}_{\theta}^{\alpha}(k) \hat{D}^{1}(k)=|k|^{\alpha-1} e^{i(\operatorname{signk})(\theta+1) \pi / 2} .
$$

\section{Conclusion}

Proposed here fractional Fick's law in three-dimensional case allows differently look at the peculiarity of energetic spectrum CR. Observed fractures in spectrum of cosmic rays ("knee" and "ankle") can be explained in the frame of fractal analysis of equation (C.5) and its multidimensional analogues.

\section{References}

[1] K.Greisen, End to the Cosmic-Ray Spectrum? Phys.Rev.Lett.,16, 748, (1966).

[2] G.T. Zatsepin, V.A. Kuzmin, Upper Limit of the Spectrum of Cosmic Rays JETP.Lett.,4, 78, (1966).

[3] Bayburina S.G.,Borisov A.S.,Cherdyntseva K.V. et.al. Nuclear Physics, B, 191, 1, (1981).

[4] Gulov Yu.A.,Ivanenko I.P.,Normuratov F., Proc. of 18th ICRC Bangalore, 5, (1983).

[5] Win M.M. et.al. J.Phys.G.Nucl.Phys. 12, 653, (1986).

[6] Lawrence M.A.,Reid PYO,Watson A.A. J.Phys.G.Nucl.Part.Phys. 17, 733, (1991).

[7] Afanasiev B.N.,et.al., Proc.Intern.Symp.on Extremly High Cosmic Rays: Astrophysics and Future Observatories Tokyo,Univer. of Tokyo, p.32, (1996).

[8] Takeda M.,et.al., Phys.Rev.Lett. 81, 1163, (1998).

[9] Bird D.Y.,et.al., Astrophys.J. 441, 144, (1995).

[10] Jui C.H.,et.al., Proc. 27th ICRC, Hamburg, V 1., (2001).

[11] Boldt E.,Chosh P., Mon.Not.R.Astron.Soc. 307, 491, (1999). 
[12] Stokes B., et.al. For the telescope Array Collab. Proc. of the Intern. Symp. of the Recent Progress of Ultra -High Energy Cosmic Ray Observation:UHECR Nagaya, Japan, December 10-12, (2010).

[13] Tinyakov P.G., et.al. For the telescope Array Collab. Proc. of the Intern. Symp. of the Recent Progress of Ultra -High Energy Cosmic Ray Observation:UHECR Nagaya, Japan, December 10-12, (2010).

[14] Tinyakov P.G., Tkachev I.I. Astropart.Phys. 24,32, (2005).

[15] Berezinsky V.,Gazizov A.Z.,Grigoreva S.I. Phys.Lett. B,612,147, (2005).

[16] Uchaikin V.V.,Zolotarev V.M., Chance and Stability, Utrecht,VSP, (1999).

[17] Feller W., An introduction to Probability Theory and its Applications, Wiley,New York,2,(1971).

[18] Gorenflo R.,Mainardi F., J.Anal.Appl. 18,(1999).

[19] P.Paradisi, R.Cesari, F.Mainardi, A.Maurizi, F.Tampieri, A generalized Fick's law to describe non-local transport effects, Physics and Chemistry of the Earth, Part B: Hydrology, Oceans and Atmosphere. 01, 3021, (2006).

[20] Samko S.G., Kilbas A.A., Marichev O.I. Fractional Integrals and Derivatives, Theory and Applications, Gordon and Breach,Amsterdam (1993). 